\title{
Rats' auditory working memory tested by continuous non-matching-to-sample performance
}

\author{
YOSHIO SAKURAI \\ Toyama Medical and Pharmaceutical University, Toyama, Japan
}

\begin{abstract}
Rats were trained in a continuous non-matching-to-sample task in order to study the retention of auditory information over a brief delay interval. A 4-sec delay period (retention interval) was imposed between trials. The subjects acquired the task with approximately 1 month of training. Subsequent changes in length of tone presentations and length of the delay period showed that response accuracy decreased with prolongation of the delay period, but did not vary as a function of tone duration. This suggests that the decrement of response accuracy with increases in delay period was due to memory decay, not to prolongation of the interval between response opportunities. Continuous non-matching-to-sample may be a useful paradigm for studying working memory, and some potential applications of the procedure are discussed.
\end{abstract}

Working memory describes a workspace or memory buffer in which information is maintained while it is being processed (Baddeley \& Hitch, 1974). A number of experimental paradigms for studying working memory have been developed (Honig, 1978), and understanding the neural bases of working memory is considered an important topic in psychobiology. Although many researchers have studied visual working memory in animals, relatively little is known about working memory in other modalities. Recently, D'Amato and Colombo (1985) and Kojima (1985) analyzed auditory working memory in the monkey, using delayed conditional discrimination or matching-to-sample tasks with auditory stimuli. In these situations, the monkeys acquired the tasks only after a relatively long period of training. Rats, on the other hand, appear to be a good species in which to investigate auditory working memory. D'Amato and Salmon (1982) directly compared monkeys with rats on discrimination of complex auditory stimuli (tunes). They found that rats actually acquired the discrimination faster than monkeys.

The delayed matching-to-sample (DMTS) paradigm offers two advantages for studying working memory. First, it minimizes the importance of peripheral mediating responses, which may occur in other delayed response tasks. Second, it permits a rigorous manipulation of stimulus parameters and interval duration, which may not be so precisely determined with delayed alternation tasks. The present study employed a version of the DMTS paradigm, the continuous non-matching-to-sample (CNM)

This research was supported by a grant-in-aid for scientific research (60710070) from the Japanese Ministry of Education, Science and Culture. A portion of the data was collected while the author was at the Department of Behavioral Sciences, Hiroshima University. The author is especially grateful to Michael J. Pontecorvo, Nova Pharmaceutical Corporation, for his careful comments on an early draft of the manuscript. The author's mailing address is Department of Psychology, Toyama Medical and Pharmaceutical University, 2630 Sugitani, Toyama 93001, Japan. task originally introduced by Pontecorvo (1983). As Pontecorvo pointed out, the CNM task shares these advantages of DMTS tasks.

Pontecorvo (1983) used visual stimuli in his original study. The present study, on the other hand, examined acquisition of CNM with auditory stimuli. In addition, the effects of varying length of tone presentation and delay interval were examined to determine whether performance decrements with increasing delay were due to memory process decay or to prolongation of the interval between response opportunities.

Preliminary reports showing neuronal correlates of CNM performance have been given elsewhere (Sakurai, 1985, 1986).

\section{METHOD}

\section{Subjects}

A total of 8 4-month-old male albino rats were obtained from Cler Japan, Inc., Osaka. The animals were individually housed, with a 12-h light/12-h dark cycle. Cage dimensions were $25 \times 15 \times 20 \mathrm{~cm}$. Lights were on from 0900 to 2100 and all behavioral testing was conducted during the light phase of the cycle. All animals were fed enough lab chow 20-30 min after their daily training session to maintain them at $80 \%$ of their ad-lib weights. Water was available continuously.

\section{Apparatus}

Behavioral training took place in a $25 \times 11 \times 35 \mathrm{~cm}$ operant chamber (Sakurai \& Sugimoto, 1985, 1986). One wall of the chamber had a $4 \times 7 \mathrm{~cm}$ translucent response panel, $3 \mathrm{~cm}$ above the floor. The panel could be illuminated by an $8-\mathrm{V}$ dc bulb and could be covered by a guillotine door. A buzzer was located behind the panel and a loudspeaker was set $45 \mathrm{~cm}$ above the top of the chamber. A food dispenser delivered a $60-\mathrm{mg}$ food pellet to a magazine located $1.5 \mathrm{~cm}$ above the floor and $1 \mathrm{~cm}$ from the corner of the right wall. The chamber was enclosed in a soundproof box (Japan Shield Enclosure, Inc.). All events were controlled and the data were recorded by microcomputer (Digital Equipment, MINC-11; Nippon Electric Corporation, PC-8801mkII). The computers were in the same room as the soundproof box. 

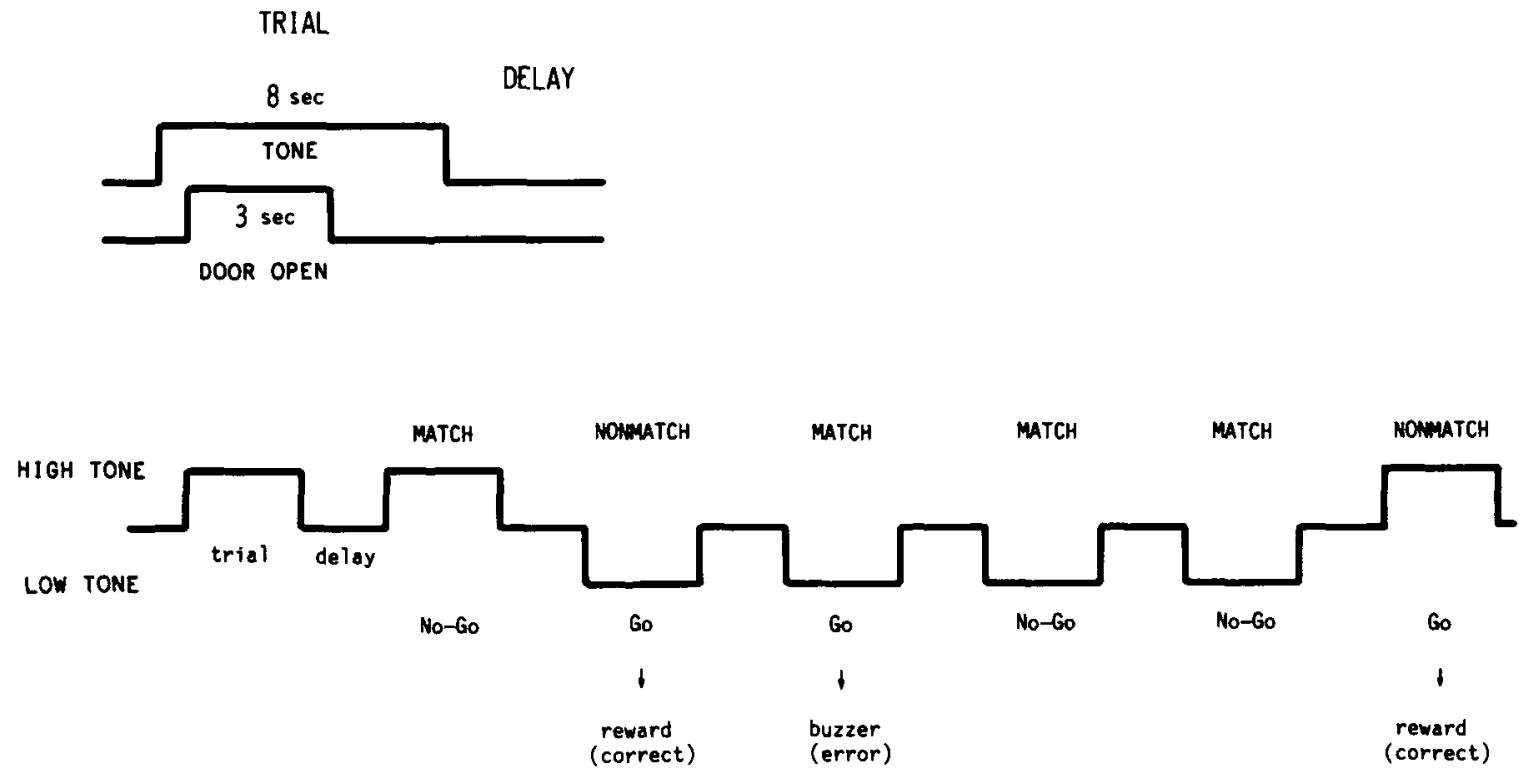

Figure 1. The upper panel portrays the sequence of events within a single CNM trial. The trial consisted of an 8-sec tone presentation and a 3-sec response opportunity period. The lower panel represents a typical sequence of trials within a session. Go responses on nonmatch trials were reinforced, whereas go responses on match trials were never reinforced and were followed by a brief buzzer presentation. The delay served as a retention interval between trials.

\section{Procedure}

All animals were given magazine training and then shaped to press the response panel for food reward. Following completion of the preliminary training, CNM training (Figure 1) was initiated. At the start of each trial, one of two tones was presented and continued for $8 \mathrm{sec}$. One second after tone onset, the guillotine door opened to make the illuminated response panel available for $3 \mathrm{sec}$. A variable number of high-tone $(8 \mathrm{kHz}, 85 \mathrm{~dB})$ trials alternated with a variable number of low-tone $(1 \mathrm{kHz}, 85 \mathrm{~dB})$ trials. The trial following a tone-stimulus change was a "nonmatch" trial. The trial following repeated stimuli was a "match" trial. Thus, nonmatches and matches were defined with respect to the preceding trial. A panelpress response (go) on a nonmatch trial turned the panel light off and was reinforced with two food pellets delivered immediately after the response. Responses on match trials also turned the panel light off, but they were never reinforced and were followed by presentation of a 0.5 -sec buzzer. An incorrect response on a match trial was always followed by two further match trials (correction trials). Contingencies on correction trials were the same as those on the initial match trial. Under this training schedule, the rat was reinforced for responding on nonmatch trials, whereas responding on match trials was extinguished. The rat thus had to remember which stimulus had been presented most recently in order to perform efficiently.

There were 30 nonmatch trials and, not counting correction trials, 90 match trials in each daily training session. On the average, there were 3 match trials between successive nonmatch trials (range, 1-5). Each stimulus (high or low tone) appeared on approximately half of the match and half of the nonmatch trials. The delay period between trials was initially 1 sec. Training continued until a rat met a criterion of $90 \%$ correct on nonmatch (go) trials and $70 \%$ correct on match (no-go) trials for 2 consecutive days. Calculation for the training criterion included neither correction trials nor the first trials (always match trials) following nonmatch trials. The delay period was incremented by $1 \mathrm{sec}$ each time the subject reached criterion level at a given delay. All rats were trained so that they acquired the task with $4 \mathrm{sec}$ of delay.
Two kinds of behavioral tests were conducted. In Test 1 , the delay was fixed at $4 \mathrm{sec}$. The period of tone presentation after the door closed (sample tone) was manipulated. Approximately equal numbers of 2-sec, 4-sec, and 8-sec sample-tone trials, not including correction trials, were randomly presented within a daily fest session. In Test 2 , the sample-tone period was fixed at $4 \mathrm{sec}$, as in the original training. The delay was randomly varied to be $2 \mathrm{sec}$, $4 \mathrm{sec}$, and $8 \mathrm{sec}$ approximately equally often per session, not including correction trials. Thus in both Test 1 and Test 2 sessions, intervals of 7,9 , or $13 \mathrm{sec}$ intervened between response opportunities. However, the retention interval varied only in Test 2 . Both Test 1 and Test 2 contained three sessions, each of which consisted of 150 trials. The order of Test 1 and Test 2 was counterbalanced among the subjects.

\section{RESULTS}

\section{Acquisition}

Figure 2 shows daily performance for the fastest (top panel), average (middle panel), and slowest (bottom panel) learners among the subjects. The rats typically exhibited a period of chance performance followed by a period of acquisition, during which correct nonmatch trials (go/nonmatch, G/NM) and/or correct match trials (no-go/match, $\mathrm{NG} / \mathrm{M}$ ) increased. The average number of daily sessions before the completion of training for all subjects was 16.2 $(S D=9.02)$ for the $1-\mathrm{sec}$ delay and $33.6(S D=13.5)$ for the 4-sec delay.

\section{Effects of Changes of Sample \\ Tone and Delay Period}

Figure 3 shows the average number of correct responses (G/NM and NG/M) during Test 1 (top panel) and Test 2 (bottom panel). Both G/NM and NG/M remained roughly 

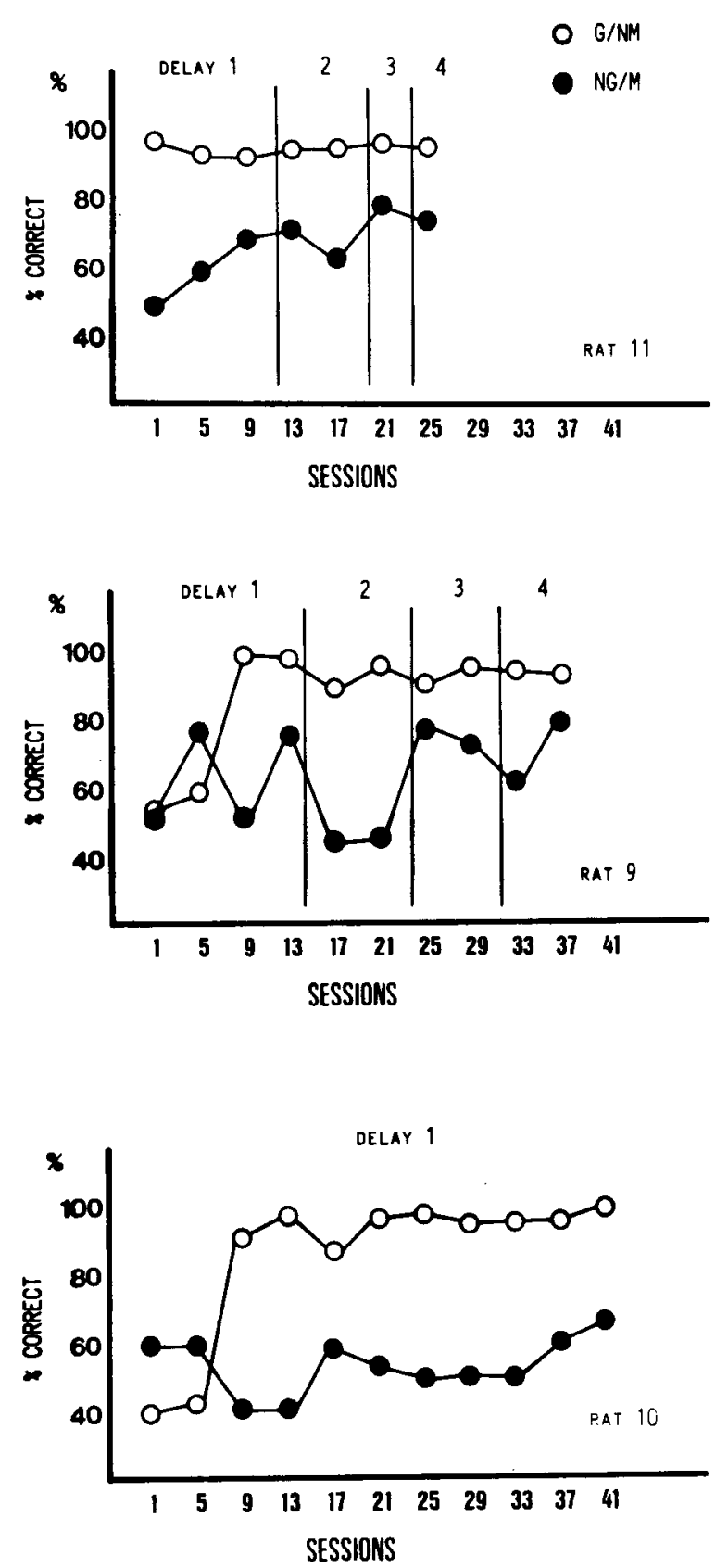

Figure 2. CNM acquisition of the fastest (top panel), average (middle panel), and slowest (bottom panel) rats with delays of $1,2,3$, and 4 sec. $G / N M=$ correct (go) nonmatch trials; $N G / M=$ correct (no-go) match trials.

constant as the sample-tone period varied among 2,4 , and $8 \mathrm{sec}$ with a 4-sec fixed delay period (Test 1 ). When the delay period was increased (Test 2), G/NM remained constant, but NG/M decreased at the 8-sec delay. Analysis of variance confirmed this decrement in correct match responses across delays $[F(2,63)=18.8, p<.01]$, and correct match responses at the 8-sec delay significantly decreased compared with the $2-\sec [t(21)=5.13$, $p<.001]$ and 4-sec $[t(21)=2.43, p<.05]$ delays.

\section{DISCUSSION}

The present results show that rats readily acquire the CNM task with auditory stimuli, and that they are capable of performing the task with a 4-sec retention period. CNM thus appears to be an appropriate task for studying auditory working memory in animals.

Some researchers (e.g., Wallace, Steinert, Scobie, \& Spear, 1980) have suggested that rats can encode and remember auditory events more efficiently than visual events. The acquisition period in the present study was actually a little shorter than that of Pontecorvo (1983), who employed visual stimuli. However, retention (change in accuracy with delay) in this study appears quite similar to that found by Pontecorvo. Moreover, rats can be further trained to achieve greater accuracy with visual stimuli (Pontecorvo \& Shores, 1986) than that seen with auditory stimuli in the present study. The present procedure allows longer sample presentation than that of Pontecorvo, and provides a fixed sample period independent of subjects' responses. According to these observations and procedural differences, better stimulus encoding or
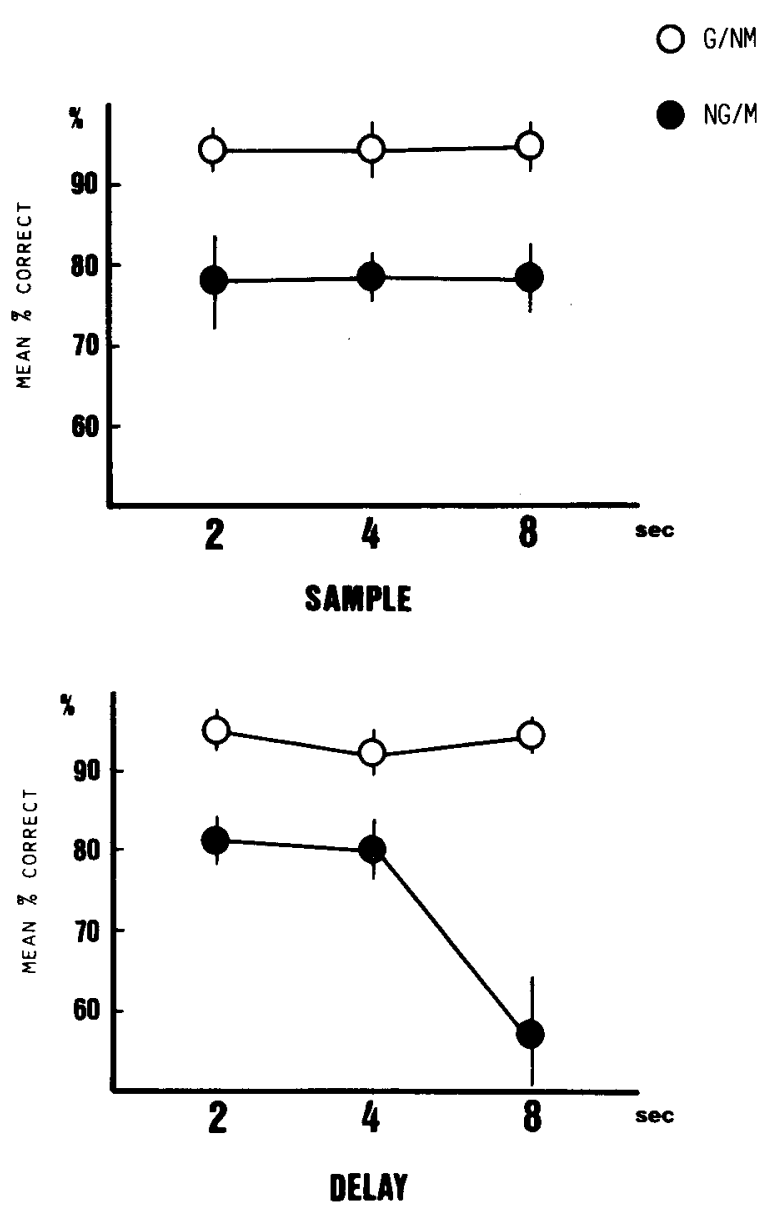

Figure 3. Mean values of correct nonmatch trials (G/NM) and correct match trials (NG/M) as a function of sample-tone period of Test 1 (upper panel) and delay period of Test 2 (lower panel). Bars show standard deviation for each mean. 
discriminability, not memory storage, is supposed to be a key difference between the present procedure and that of Pontecorvo. It is difficult to directly compare efficiency of memory for auditory and visual events in these studies.

Because the interresponse interval (IRI) comprises the duration of the sample tone (the tone presentation after the response opportunity) plus the delay period, the IRI varied equally in Tests 1 and 2 . The performance decrement shown only in Test 2 , therefore, cannot be attributed to an increased tendency to respond induced by a prolonged IRI (Pontecorvo, 1983; Roberts \& Kraemer, 1982). Some memory factors, for example, trace decay (e.g., Roberts, 1972), may have caused the decrement of correct performance in Test 2 . Test 1 , on the other hand, showed stable performance with changes in sample duration. That result seems to be inconsistent with studies reporting that forgetting of events was inversely related to the duration of stimulus exposure (e.g., Nelson \& Wasserman, 1978). The relatively long stimulus presentation $(8 \mathrm{sec})$ in the present study may be the reason why the changes in length of such stimulus presentation (sample duration 2-8 sec) had no effect on correct performance.

Although no-go performance changed according to the working-memory decay in the CNM task, another type of correct performance, go performance on nonmatch trials, was stable against the prolongation of delay. Pontecorvo (1983) reported similar results. When only the go response was reinforced in a go/no-go task, as in the present experiment, the subject could keep readiness to respond (go) throughout the training session. Therefore, go was very apt to occur and was very consistent throughout the session. On the other hand, no-go is a deviation from that basic tendency to respond, which may be why correct no-go was relatively unstable and easily changed along variation of the delay period. This type of no-go response, which has no reinforcement contingency, is not an active operant response (Anger, 1983). The mechanism underlying the releasing of a reinforcement-contingent nogo response may be different from that underlying a nonreinforcement-contingent no-go response.

The present CNM paradigm offers some advantages for studying the neural processes underlying working memory. First, the CNM task can permit rigorous definition and manipulation of the to-be-remembered stimulus. Most previous studies using rats have employed spatial discrimination or alternation (e.g., Grant, 1982) as tasks with delay. However, in these tasks it is not clear which types of stimulus the animal remembers. Because different neural structures in the brain may underlie different types of memory (e.g., Squire \& Zola-Morgan, 1983), it is at least necessary to define types of to-be-remembered stimuli in psychobiological studies of memory. Second, the present CNM task requires auditory memory processes, on which few psychobiological studies have been carried out. According to many studies using visual events, the frontal cortex, hippocampus, and/or thalamus have been supposed in general to be involved in memory processes (e.g., Mishkin, 1982). But these areas should be further examined to determine whether they are also related to memory for auditory events and for visual events. A third advantage is that the delay period of the CNM task really functions as a retention interval, because only the prolongation of the delay produces the decrement in correct performance. In investigation of memoryrelated neurons or structures, analysis of neuronal correlates and behavioral changes with manipulation of retention interval is a useful strategy (e.g., Kojima \& GoldmanRakic, 1982). Finally, the training period for the CNM task is rather short compared with that for the DMTStype tasks, and rats can be used as subjects. Because of their genetic purity, their evolutional importance, and the great amount of knowledge about their behavior, rats have been and no doubt will continue to be the most popular subjects in all psychobiological fields, from the molecular to the behavioral level.

\section{REFERENCES}

ANGER, D. (1983). Reinforcement of inhibition. Journal of the Experimental Analysis of Behavior, 39, 213-226.

BadDEley, A. D., \& Hitch, G. J. (1974). Working memory. In G. A. Bower (Ed.), The psychology of learning and motivation: Advances in research and theory (Vol. 8, pp. 47-90). New York: Academic Press.

D'Aмato, M. R., \& Colombo, M. (1985). Auditory matching-to-sample in monkeys (Cebus apella). Animal Leaming \& Behavior, 13, 375-382.

D'Amato, M. R., \& SAlmon, D. P. (1982). Tune discrimination in monkeys (Cebus apella) and in rats. Animal Learning \& Behavior, 10, 126-134.

GraNT, D. S. (1982). Stimulus control and information processing in rat short-term memory. Joumal of Experimental Psychology: Animal Behavior Process, 8, 154-164.

HoNiG, W. K. (1978). Studies of working memory in the pigeon. In S. H. Hulse, H. Fowler, \& W. K. Honig (Eds.), Cognitive processes in animal behavior (pp. 211-248). Hillsdale, NJ: Erlbaum.

Колма, S. (1985). Auditory short-term memory in the Japanese monkey. International Journal of Neuroscience, 25, 255-262.

KoJma, S., \& Goldman-Rakic, P. S. (1982). Delayed-related activity of prefrontal neurons in rhesus monkeys performing delayed response. Brain Research, 248, 43-49.

Mishkin, M. (1982). A memory system in the monkey. Philosophical Transactions of the Royal Society of London, B 298, 85-95.

Nelson, K. R., \& Wasserman, E. A. (1978). Temporal factors influencing the pigeon's successive matching-to-sample performance: Sample duration, intertrial interval and retention. Journal of the Experimental Analysis of Behavior, 30, 153-167.

PonTEConvo, M. J. (1983). Effects of proactive interference on rats' continuous nonmatching-to-sample performance. Animal Learning \& Behavior, 11, 356-366.

Pontecorvo, M. J., \& SHOREs, E. I. (1986). Effects of scopolamine and propranalol on rats' two choice continuous non-matching to sample memory performance. Neuroscience Abstracts, 12, 714.

RoBerTs, W. A. (1972). Short-term memory in the pigeon: Effects of repetition and spacing. Joumal of Experimental Psychology, 94, 74-83.

RoberTs, W. A., \& KAEMER, P. J. (1982). Some observations on the effects of intertrial interval and delay on delayed matching to sample in pigeons. Journal of Experimental Psychology: Animal Behavior Processes, 8, 342-353.

SAKURAI, Y. (1985). Neuronal activity of prefrontal cortex and dorso- 
medial thalamus during a continuous nonmatching-to-sample task in the rat. Neuroscience Abstracts, 11, 526.

SAKURAI, Y. (1986). Neuronal activities in the rat prefrontal cortex and dorsomedial thalamus related to the working memory process for tone. Neuroscience Research, Suppl. 3, S12.

SaKURAI, Y., \& SUGimoto, S. (1985). Effects of lesions of prefrontal cortex and dorsomedial thalamus on delayed go/no-go alternation in rats. Behavioral Brain Research, 17, 213-219.

SAKURAI, Y., \& SugrmoTo, S. (1986). Mutiple unit activity of prefrontal cortex and dorsomedial thalamus during delayed go/no-go alternation in the rat. Behavioral Brain Research, 20, 295-301.

SQUiRE, L. R., \&ola-Morgan, S. (1983). The neurology of memory: The case for correspondence between the findings for human and nonhuman primate. In J. A. Deutsch (Ed.) The physiological basis of memory (2nd ed., pp. 199-268). London: Academic Press.

Wallace, J., Steinert, P. A., Scobie, S. R., \& Spear, N. E. (1980). Stimulus modality and short-term memory in rats. Animal Learning \& Behavior, 8, 10-16.

(Manuscript received April 9, 1987;

revision accepted for publication July $27,1987$. ) 